NBI-HE-93-57

October 5,1993

\title{
Wilson loop on a sphere
}

\author{
D.V. Boulatov \\ The Niels Bohr Institute \\ University of Copenhagen \\ Blegdamsvej 17, 2100 Copenhagen $\varnothing$ \\ Denmark
}

\begin{abstract}
We give the formula for a simple Wilson loop on a sphere which is valid for an arbitrary $\mathrm{QCD}_{2}$ saddle-point $\rho(x): W\left(A_{1}, A_{2}\right)=\oint \frac{d x}{2 \pi i} \exp \left(\int d y \frac{\rho(y)}{y-x}+A_{2} x\right)$. The strong-coupling-phase solution is investigated.
\end{abstract}




\section{Introduction}

$Q C D_{2}$ has recently drawn much attention because of the new insights brought about by the Gross-Taylor reinterpretation of its partition function as the sum over branched coverings [1]. The similar interpretation exists for Wilson loop operators on the plane as well [2]. The topological nature of $Q C D_{2}$ was noticed long ago by A.A.Migdal [3] who proposed to use a character expansion of Boltzman weights to manifest it. One can always integrate over a link variable by using only the orthogonality of group elements. Following this guideline, the partition function of $Q C D_{2}$ on an arbitrary 2-manifold was obtained in ref. [4] in the form of a sum over irreps of a gauge group. Recently, on the sphere, the third order phase transition with respect to the area was found [5] (see also [6], where the weak coupling partition function was calculated). Wilson loop operators on the plane were investigated in refs. [7] and shown to display stringy features, in particular, to obey the area low. However, in the weak coupling phase on the sphere their behavior is completely different [5]. In the present paper, we give the general solution for a simple loop establishing a bridge between results of refs. [7] and [5]. Having known the answer, non-trivial Wilson loops can be found by solving the renormalized Makeenko-Migdal equation adopted for the sphere [8].

For reader's convenience, let us repeat known results from Refs. [由, 5, 6] which we shall need later. It will also allow us to introduce a notation.

If the Boltzmann weight, as a function of a holonomy along a plaquette, is taken in the form of the $U(N)$ heat kernel, it is reproduced after the integration over internal gauge variables, and one can easily obtain the partition function for a disc (as a function of an area, $A$, and a holonomy along the boundary, $U$ ) in the form of the sum over $U(N)$ representations

$$
Z_{1}\left(g^{2} A, U\right)=\sum_{R} d_{R} \chi_{R}(U) \exp \left(-\frac{g^{2} A}{2 N} C_{R}\right)
$$

where $\chi_{R}(U)$ is the character of an $R$ 'th irrep; $d_{R}=\chi_{R}(I)$ is its dimension:

$$
d_{R}=\prod_{1 \leq i<j \leq N}\left(1+\frac{n_{i}-n_{j}}{j-i}\right)
$$

$C_{R}$ is the value of the second Casimir operator: 


$$
C_{R}=\sum_{i=1}^{N} n_{i}\left(n_{i}+N+1-2 i\right)
$$

where $n_{i}$ are highest weight components of $R, R \equiv\left[n_{1}, \ldots, n_{N}\right]$, i.e. the numbers of boxes in rows of the Young table $\left(n_{1} \geq n_{2} \geq \ldots \geq n_{N}\right)$. We are considering $U(N)$, therefore $n_{N}$ is unrestricted.

The gauge coupling constant $g^{2}$ can be absorbed into the area, $A$, (in what follows, we put $g^{2}=1$ ). For $A=0$, Eq. (1) becomes the group $\delta$-function: $Z_{1}(0, U)=\delta(U, 1)$.

The partition function for a closed surface of a genus $p$ can be represented as the sum [4]:

$$
Z_{p}(A)=\sum_{R} d^{2(1-p)} \exp \left(-\frac{A}{2 N} C_{R}\right)
$$

In the spherical case, $p=0$, the sum becomes divergent at small areas. Hence, in the large $N$ limit, a non-trivial saddle-point should exist. In this case we can introduce the continuous function [6]

$$
h(x)=\lim _{N \rightarrow \infty} \frac{1}{N}\left(i-\frac{N}{2}-n_{i}\right) ; \quad x=\frac{i}{N}-\frac{1}{2}
$$

then the saddle-point equation takes the well known form

$$
\frac{A}{2} h=f d y \frac{\rho(y)}{h-y}
$$

Here,

$$
\rho(h)=\frac{\partial x}{\partial h}
$$

obeys the inequality

$$
\rho(h) \leq 1
$$

If Eq. (8) is ignored, the solution to Eq. (6) is the well known semi-circle distribution

$$
\rho(h)=\frac{1}{\pi} \sqrt{A-\frac{A^{2} h^{2}}{4}}
$$


which is obviously valid for small areas: $A<\pi^{2}$. For larger areas $\left(A>\pi^{2}\right)$, the inequality (8) is crucial and Douglas and Kazakov [5] have found in this case for the function

$$
f(z)=\int d y \frac{\rho(y)}{z-y}
$$

the following answer

$$
\begin{aligned}
f(z)= & \frac{A}{2} z+\frac{1}{z} \int_{-b}^{+b} \frac{d y}{1-\frac{y^{2}}{z^{2}}} \sqrt{\frac{\left(a^{2}-z^{2}\right)\left(b^{2}-z^{2}\right)}{\left(a^{2}-y^{2}\right)\left(b^{2}-y^{2}\right)}}= \\
& \frac{A}{2} z+\frac{2}{a z} \sqrt{\left(a^{2}-z^{2}\right)\left(b^{2}-z^{2}\right)} \Pi_{1}\left(-\frac{b^{2}}{z^{2}}, \frac{b}{a}\right)
\end{aligned}
$$

where $\Pi_{1}(x, k)$ is the complete elliptic integral of the third kind with the modulus $k=\frac{b}{a}$. The density is the imaginary part of this function on the cut, $\rho(z)=\frac{1}{\pi} \operatorname{Im} f(z)$, and has the form

$$
\rho(z)= \begin{cases}-\frac{2}{\pi a z} \sqrt{\left(a^{2}-z^{2}\right)\left(z^{2}-b^{2}\right)} \Pi_{1}\left(-\frac{b^{2}}{z^{2}}, \frac{b}{a}\right) & \text { for }-a<z<-b \\ 1 & \text { for }-b<z<b \\ \frac{2}{\pi a z} \sqrt{\left(a^{2}-z^{2}\right)\left(z^{2}-b^{2}\right)} \Pi_{1}\left(-\frac{b^{2}}{z^{2}}, \frac{b}{a}\right) & \text { for } b<z<a\end{cases}
$$

The parameters are to be determined from the equations"

$$
a\left(2 E-k^{\prime 2} K\right)=1 \quad a A=4 K
$$

At the critical value $A_{c}=\pi^{2}$, a third order phase transition takes place.

Another interesting quantity is the Wilson loop average on a sphere:

$$
\begin{aligned}
W\left(A_{1}, A_{2}\right)= & \frac{1}{Z_{0}\left(A_{1}+A_{2}\right)} \int d U Z_{1}\left(A_{1}, U\right) Z_{1}\left(A_{2}, U^{+}\right) \frac{1}{N} \operatorname{tr} U= \\
& \frac{1}{N Z_{0}\left(A_{1}+A_{2}\right)} \sum_{R, S} d_{R} d_{S}\langle R, f \mid S\rangle \exp \left(-\frac{A_{1}}{2 N} C_{R}-\frac{A_{2}}{2 N} C_{S}\right)
\end{aligned}
$$

\footnotetext{
${ }^{1}$ Working with elliptic functions we always use conventions adopted in the book [9].
} 
where $\langle R, f \mid S\rangle=0$ or 1 is the multiplicity of an irrep $S$ in the tensor product of $R$ with the fundamental representation $f$. Douglas and Kazakov [5] gave for $W$ the expression

$$
W\left(A_{1}, A_{2}\right)=\sqrt{\frac{A_{1}+A_{2}}{A_{1} A_{2}}} J_{1}\left(\sqrt{\frac{4 A_{1} A_{2}}{A_{1}+A_{2}}}\right)
$$

valid in the weak coupling (small areas) phase and wrote after that "It would be very interesting to calculate the Wilson loop in the strong coupling phase", which is our goal in the present paper.

\section{Wilson loop for an arbitrary Young table}

If the sum over irreps is dominated by a particular representation $R$, Eq. (14) can be written as

$$
W\left(A_{1}, A_{2}\right)=\frac{1}{N} \sum_{S} \frac{d_{S}}{d_{R}}\langle R, f \mid S\rangle \exp \left(-\frac{A_{2}}{2 N}\left(C_{S}-C_{R}\right)\right)
$$

Obviously, $W\left(A_{1}, 0\right)=1$, because of the identity

$$
\frac{1}{N} \sum_{S} \frac{d_{S}}{d_{R}}\langle R, f \mid S\rangle=1
$$

The equality $W\left(0, A_{2}\right)=1$ is less trivial in this non-symmetric representation and should hold dynamically (i.e. depending on an actual form of the saddlepoint $R$ ). If the number of boxes in the Young table of $R$ is of the order $N^{2}$, the formula (16) is really symmetric. However, if the saddle-point is dominated by the trivial representation, but $S=0$ and $R=\bar{f}$, it is obviously incorrect, because $Z_{0}=1$. Nevertheless, our final answer will be free from this loop-hole.

The sum in Eq. (16) goes over all Young tables $S$ which can be obtained from the one for the saddle-point $R$ by adding one box. It is possible in rows $i$ where $n_{i}<n_{i-1}$. Therefore, we can introduce the density of vacancies

$$
\eta(h)= \begin{cases}1 & \text { if } \rho(h)<\frac{1}{2} \\ 1-\rho(h) & \text { if } \frac{1}{2}<\rho(h)<1\end{cases}
$$


and, like it was done in Eq. (6) and (7), rewrite Eq. (16) as the integral

$$
W\left(A_{1}, A_{2}\right)=\int d h \eta(h) D(h) e^{A_{2} h}
$$

where we have used that, for Young tables of $S$ and $R$ differing by one box in the $k^{\prime}$ th row,

$$
\frac{1}{N}\left(C_{S}-C_{R}\right)=\frac{1}{N}\left(2 n_{k}+N+1-2 k\right) \rightarrow-2 h(x)
$$

$D(h)$ is the large $N$ limit of $d_{S} / d_{R}$ :

$$
D\left(\frac{k}{N}-\frac{1}{2}\right)=\frac{\prod_{i \neq k}\left(n_{k}+1-k-n_{i}+i\right)}{\prod_{i \neq k}\left(n_{k}-k-n_{i}+i\right)}=\exp \sum_{i \neq k} \log \left(1-\frac{1 / N}{h\left(\frac{k}{N}-\frac{1}{2}\right)-h\left(\frac{i}{N}-\frac{1}{2}\right)}\right)
$$

We should be accurate in calculating the sum in the exponent. Let $M=O(\sqrt{N})$. If $\rho(h)<\frac{1}{2}$, we can substitute, for $|i-k|<M$,

$$
N\left[h\left(\frac{i}{N}-\frac{1}{2}\right)-h\left(\frac{k}{N}-\frac{1}{2}\right)\right] \approx h^{\prime}\left(\frac{k}{N}-\frac{1}{2}\right)(i-k)
$$

Then the sum takes the form

$$
\begin{aligned}
& \log D\left(\frac{k}{N}-\frac{1}{2}\right)=\left(\sum_{|i-k|>M}+\sum_{|i-k|<M}\right) \log \left(1+\frac{1 / N}{h\left(\frac{i}{N}-\frac{1}{2}\right)-h\left(\frac{k}{N}-\frac{1}{2}\right)}\right) \approx \\
& \frac{1}{N} \sum_{|i-k|>M} \frac{1}{h\left(\frac{i}{N}-\frac{1}{2}\right)-h\left(\frac{k}{N}-\frac{1}{2}\right)}+\sum_{|i-k|<M} \log \left(1+\frac{1}{h^{\prime}\left(\frac{k}{N}-\frac{1}{2}\right)(i-k)}\right) \rightarrow \\
& f d y \frac{\rho(y)}{y-h}+\sum_{n=1}^{\infty} \frac{[-\rho(h)]^{n}}{n} \sum_{q=1}^{\infty} \frac{1+(-1)^{q}}{q^{n}}=f d y \frac{\rho(y)}{y-h}+\log \left(\frac{\sin \pi \rho(h)}{\pi \rho(h)}\right)
\end{aligned}
$$

If $\frac{1}{2}<\rho(h)<1$, the analysis has to be more careful. In this case, the product goes actually over columns of the Young table and there are a lot of cancellations between the numerator and the denominator in Eq. (21). Let $\left(j_{1} \equiv 1, j_{2}, \ldots, j_{m}, j_{m+1} \equiv N+1\right)$ be a sequence of indices such that $n_{j_{k}-1}-n_{j_{k}}=1$ and $n_{j_{k}-2}-n_{j_{k}-1}=0$, then we have

$$
D\left(\frac{j_{k}}{N}-\frac{1}{2}\right)=\left(j_{k+1}-j_{k}\right) \prod_{s \neq k} \frac{j_{s+1}-j_{k}+s-k}{j_{s}-j_{k}+s-k}
$$


In this case, if $|s-k|<M$, we can expand $j_{s}-j_{k} \approx-\frac{\rho(h)}{1-\rho(h)}(s-k)$ and analogously to Eq. (23) find

$$
\begin{gathered}
\log D\left(\frac{j_{k}}{N}-\frac{1}{2}\right) \approx \frac{1}{N} \sum_{|i-k|>j_{k+M}-j_{k}} \frac{1}{h\left(\frac{i}{N}-\frac{1}{2}\right)-h\left(\frac{j_{k}}{N}-\frac{1}{2}\right)}+ \\
\sum_{|s-k|<M} \log \left(1+\frac{\rho(h)}{s-k}\right) \rightarrow f d y \frac{\rho(y)}{y-h}+\log \left(\frac{\sin \pi \rho(h)}{\pi \rho(h)}\right)
\end{gathered}
$$

The factor $j_{k+1}-j_{k} \rightarrow \frac{\rho(h)}{1-\rho(h)}$ in Eq. (24) combines with the density of vacancies (18) and we find the solution which is valid for an arbitrary $\rho(h)$

$$
W\left(A_{1}, A_{2}\right)=\int \frac{d h}{\pi} \sin \pi \rho(h) \exp \left(-f d y \frac{\rho(y)}{h-y}+A_{2} h\right)
$$

This formula can be nicely represented as the contour integral

$$
W\left(A_{1}, A_{2}\right)=\oint_{C} \frac{d h}{2 \pi i} e^{A_{2} h-f(h)}
$$

where $f(h)$ is defined in Eq. (10) and the contour $C$ encircles the cut of $f(h)$. Obviously $W\left(A_{1}, 0\right)=1$ : to prove it one has to expand the exponential in powers of $f(h)$ and take the residue at the infinity. On the other hand, owing to the saddle-point equation (6), Eq. (26) can be rewritten as

$$
W\left(A_{1}, A_{2}\right)=\int \frac{d h}{\pi} \sin \pi \rho(h) e^{\frac{A_{2}-A_{1}}{2} h}=\oint_{\bar{C}} \frac{d h}{2 \pi i} e^{-A_{1} h+f(h)}
$$

where the contour $\bar{C}$ encircles the cut of $f(h)$ in the negative direction. Here, $W\left(0, A_{2}\right)=1$ is obvious.

\section{$3 \quad$ Particular cases}

In the weak coupling phase, $f(h)=\frac{A}{2} h-\sqrt{\frac{A^{2} h^{2}}{4}-A}\left(A=A_{1}+A_{2}\right)$, and it is convenient to introduce the variable $z=\frac{\sqrt{A}}{i f}$ in terms of which the integral (27) takes the form

$$
W_{\mathrm{wc}}\left(A_{1}, A_{2}\right)=\frac{1}{i \sqrt{A}} \oint_{C} \frac{d z}{2 \pi i}\left(1+\frac{1}{z^{2}}\right) e^{i a z+i \frac{b}{z}}
$$


where $a=\frac{1}{2} \sqrt{A}-\frac{A_{1}-A_{2}}{2 \sqrt{A}}$ and $b=\frac{1}{2} \sqrt{A}+\frac{A_{1}-A_{2}}{2 \sqrt{A}}$. Expanding the exponential and taking the residue at zero, we find

$$
\begin{gathered}
W_{\mathrm{wc}}\left(A_{1}, A_{2}\right)=\frac{1}{i \sqrt{A}} \oint_{C} \frac{d z}{2 \pi i}\left(1+\frac{1}{z^{2}}\right) \sum_{n=0}^{\infty} \frac{i^{2 n+1}}{n !(n+1) !}\left(a^{n+1} b^{n} z+a^{n} b^{n+1} \frac{1}{z}\right)= \\
\sum_{n=0}^{\infty} \frac{(-1)^{n}}{n !(n+1) !}(a b)^{n}=\sqrt{\frac{A_{1}+A_{2}}{A_{1} A_{2}}} J_{1}\left(\sqrt{\frac{4 A_{1} A_{2}}{A_{1}+A_{2}}}\right)
\end{gathered}
$$

So, we have reproduced the formula (15).

In the strong coupling phase, we have to use the Douglas-Kazakov solution (11). It is convenient to change the variable

$$
h=b \operatorname{sn} u
$$

then we have

$$
f(b \operatorname{sn} u)=\frac{b A}{2} \operatorname{sn} u-2 K Z(u)
$$

where

$$
Z(u)=\frac{\partial}{\partial u} \log \theta_{4}\left(\frac{u}{2 K}\right)=\frac{4 \pi}{2 K} \sum_{m=1}^{\infty} \frac{q^{m}}{1-q^{2 m}} \sin \frac{m \pi u}{K}
$$

is one of classical Jacobi's functions [9]. It is not double periodic

$$
Z(u+2 K)=Z(u) \quad Z\left(u+i 2 K^{\prime}\right)=Z(u)-\frac{i \pi}{K}
$$

However, we have the combination $\exp \{2 K Z(u)\}$ which is elliptic. Therefore, the contour integral can be reduced to a residue at $u=i K^{\prime}$ (which is mapped to the infinity by the function (31)).

$$
W_{\mathrm{sc}}\left(A_{1}, A_{2}\right)=b \oint_{C^{\prime}} \frac{d u}{2 \pi i} \operatorname{cn} u \operatorname{dn} u e^{\frac{A_{2}-A_{1}}{2} b \operatorname{sn} u+2 K Z(u)}
$$

Let us notice that

$$
\frac{A b}{2} \operatorname{sn} u-2 K Z(u)=-4 \pi \sum_{m=1}^{\infty}(-1)^{m} \frac{q^{\frac{m}{2}}}{1-q^{m}} \sin \frac{m \pi u}{2 K}=-\frac{\theta_{3}^{\prime}\left(\frac{u}{4 K} \mid \frac{\tau}{2}\right)}{\theta_{3}\left(\frac{u}{4 K} \mid \frac{\tau}{2}\right)}
$$


and

$$
\frac{A b}{2} \operatorname{sn} u+2 K Z(u)=4 \pi \sum_{m=1}^{\infty} \frac{q^{\frac{m}{2}}}{1-q^{m}} \sin \frac{m \pi u}{2 K}=\frac{\theta_{4}^{\prime}\left(\frac{u}{4 K} \mid \frac{\tau}{2}\right)}{\theta_{4}\left(\frac{u}{4 K} \mid \frac{\tau}{2}\right)}
$$

where $\tau=i \frac{K^{\prime}}{K}$ and $q=e^{i \pi \tau}$. For convenience, let us shift the variable $u=z+i K^{\prime}$, then

$$
\begin{gathered}
\operatorname{cn}\left(z+i K^{\prime}\right)=\frac{\mathrm{dn} z}{i k \operatorname{sn} z} \quad \operatorname{dn}\left(z+i K^{\prime}\right)=\frac{\operatorname{cn} z}{i \sin z} \\
4 K \frac{\partial}{\partial z} \log \theta_{3}\left(\frac{z+i K^{\prime}}{4 K} \mid \frac{\tau}{2}\right)=4 K \frac{\partial}{\partial z} \log \theta_{2}\left(\frac{z}{4 K} \mid \frac{\tau}{2}\right)-i \pi \stackrel{\text { def }}{=} \phi_{2}(z)-i \pi
\end{gathered}
$$

and

$$
4 K \frac{\partial}{\partial z} \log \theta_{4}\left(\frac{z+i K^{\prime}}{4 K} \mid \frac{\tau}{2}\right)=4 K \frac{\partial}{\partial z} \log \theta_{1}\left(\frac{z}{4 K} \mid \frac{\tau}{2}\right)-i \pi \stackrel{\text { def }}{=} \phi_{1}(z)-i \pi
$$

Now we are able to write Eq. (27) in the strong coupling phase as the integral

$$
W_{\mathrm{sc}}\left(A_{1}, A_{2}\right)=-a \oint_{O} \frac{d z}{2 \pi i} \frac{\mathrm{cn} z \mathrm{dn} z}{\mathrm{sn}^{2} z} e^{\frac{A_{1}}{A} \phi_{2}(z)+\frac{A_{2}}{A} \phi_{1}(z)}
$$

where the contour $O$ encircles zero in the positive direction. Let us give the Fourier expansions of $\phi_{1}$ and $\phi_{2}$ :

$$
\begin{gathered}
\phi_{1}(z)=\pi \cot \frac{\pi z}{4 K}+4 \pi \sum_{m=1}^{\infty} \frac{q^{m}}{1-q^{m}} \sin \frac{m \pi z}{2 K} \\
\phi_{2}(z)=-\pi \tan \frac{\pi z}{4 K}+4 \pi \sum_{m=1}^{\infty}(-1)^{m} \frac{q^{m}}{1-q^{m}} \sin \frac{m \pi z}{2 K}
\end{gathered}
$$

The Riemann surface of $f(h)$ has two sheets and, therefore, two points in every mesh on the $z$-plane correspond to the infinity of the $h$-plane $(4 K n+$ $i 2 K^{\prime} m$ and $\left.4 K\left(n+\frac{1}{2}\right)+i 2 K^{\prime} m\right)$. We can as well shrink the contour on the second sheet or, equivalently, around the point $2 K$. As $\phi_{1}(z+2 K)=\phi_{2}(z)$ and $\phi_{2}(z+2 K)=\phi_{1}(z)$, we find that $W\left(A_{1}, A_{2}\right)=W\left(A_{2}, A_{1}\right)$ holds in the strong coupling phase as well. 
The representation (41) is convenient for the investigation of the transition point, $A=\pi^{2}$, which corresponds to the limit $k \rightarrow 0, K^{\prime} \rightarrow \infty, K \rightarrow \frac{\pi}{2}$ when

$$
\operatorname{sn} z \rightarrow \sin z \quad \text { cn } z \rightarrow \cos z \quad \operatorname{dn} z \rightarrow 1
$$

and we find

$$
\begin{gathered}
W_{\mathrm{sc}}\left(A_{1}, \pi^{2}-A_{1}\right)=-\frac{2}{\pi} \oint_{O} \frac{d z}{2 \pi i} \frac{\cos z}{\sin ^{2} z} e^{-\frac{A_{1}}{\pi} \tan \frac{z}{2}+\left(\pi-\frac{A_{1}}{\pi}\right) \cot \frac{z}{2}}= \\
\frac{1}{\pi} \oint_{O} \frac{d x}{2 \pi i}\left(1-\frac{1}{x^{2}}\right) e^{-\frac{A_{1}}{\pi} x+\left(1-\frac{A_{1}}{\pi}\right) \frac{1}{x}}=\sum_{n=0}^{\infty} \frac{(-1)^{n}}{n !(n+1) !}\left(\frac{A_{1}\left(\pi^{2}-A_{1}\right)}{\pi^{2}}\right)^{n} \\
=\frac{\pi^{2}}{A_{1}\left(\pi^{2}-A_{1}\right)} J_{1}\left(2 \sqrt{A_{1}\left(1-A_{1} / \pi^{2}\right)}\right)
\end{gathered}
$$

which coincides with the weak coupling solution at the transition point.

The Wilson loop on the plane $\left(A_{1} \rightarrow \infty, A_{2}\right.$ finite $)$ corresponds to the limit $k \rightarrow 1, K^{\prime} \rightarrow \frac{\pi}{2}, K \rightarrow \infty$. Here, it is convenient to make the modular transformation $\tilde{k}=k^{\prime}, \tilde{k^{\prime}}=k, \widetilde{K}=K^{\prime}, \widetilde{K^{\prime}}=K, z=-i u$ after which

$$
\operatorname{sn}(z, k)=\frac{\operatorname{sn}\left(u, k^{\prime}\right)}{i \operatorname{cn}\left(u, k^{\prime}\right)} \quad \operatorname{cn}(z, k)=\frac{1}{\operatorname{cn}\left(u, k^{\prime}\right)} \quad \operatorname{dn}(z, k)=\frac{\operatorname{dn}\left(u, k^{\prime}\right)}{\operatorname{cn}\left(u, k^{\prime}\right)}
$$

and

$$
\begin{aligned}
& \phi_{1}(z)=4 K i \frac{\partial}{\partial u} \log \theta_{1}\left(\frac{u}{2 K^{\prime}} \frac{1}{2 \tilde{\tau}} \mid-\frac{1}{2 \tilde{\tau}}\right)=4 K i \frac{\partial}{\partial u} \log \theta_{1}\left(\frac{u}{2 K^{\prime}} \mid 2 \tilde{\tau}\right)+i \frac{\pi u}{K^{\prime}} \\
& \phi_{2}(z)=4 K i \frac{\partial}{\partial u} \log \theta_{2}\left(\frac{u}{2 K^{\prime}} \frac{1}{2 \tilde{\tau}} \mid-\frac{1}{2 \tilde{\tau}}\right)=4 K i \frac{\partial}{\partial u} \log \theta_{4}\left(\frac{u}{2 K^{\prime}} \mid 2 \tilde{\tau}\right)+i \frac{\pi u}{K^{\prime}}
\end{aligned}
$$

where $\tilde{\tau}=i \frac{K}{K^{\prime}}$. After the modular transformation the integral takes the form

$$
W_{\mathrm{sc}}\left(A_{1}, A_{2}\right)=-a i \oint_{O} \frac{d u}{2 \pi i} \frac{\operatorname{dn}\left(u, k^{\prime}\right)}{\operatorname{sn}^{2}\left(u, k^{\prime}\right)} e^{\frac{2 i K A_{1}}{K^{\prime} A} \tilde{\phi}_{4}(u)+\frac{2 i K A_{2}}{K^{\prime} A} \tilde{\phi}_{1}(u)+i \frac{\pi u}{K^{\prime}}}
$$

where $\tilde{\phi}_{n}(u)=2 K^{\prime} \frac{\partial}{\partial u} \log \theta_{n}\left(\frac{u}{2 K^{\prime}} \mid 2 \tilde{\tau}\right)$.

The natural expansion parameter here is

$$
\tilde{q} \equiv e^{i \pi \tilde{\tau}}=\exp \left(-\frac{\pi a A}{4 K^{\prime}}\right) \rightarrow e^{-\frac{A}{4}}
$$


and, to perform the expansion, it is convenient to reexpress all quantities involved through the theta functions:

$$
\begin{aligned}
& \frac{2 K^{\prime}}{\pi}=\theta_{3}^{2}(0)=1+4 \tilde{q}+4 \tilde{q}^{2}+\ldots \\
& \frac{2 K^{\prime}}{\pi} \frac{\operatorname{dn}\left(2 K^{\prime} v, k^{\prime}\right)}{\operatorname{sn}^{2}\left(2 K^{\prime} v, k^{\prime}\right)}=\frac{\theta_{2}^{2}(0) \theta_{4}(0)}{\theta_{3}(0)} \frac{\theta_{3}(v) \theta_{4}(v)}{\theta_{1}^{2}(v)}=\frac{1}{\sin ^{2} \pi v}\left[1-4 \tilde{q}-4 \tilde{q}^{2}\left(\cos 2 \pi v-\sin ^{2} 2 \pi v\right)+\ldots\right] \\
& a=\frac{1}{2 E-k^{\prime 2} K}=\frac{1}{2\left(1-4 \tilde{q}+12 \tilde{q}^{2}-8 \tilde{q}^{2} \log \tilde{q}+\ldots\right)} \\
& \tilde{\phi}_{1}\left(2 K^{\prime} v\right)=\pi \cot \pi v+4 \pi \tilde{q}^{4} \sin 2 \pi v+\ldots \\
& \tilde{\phi}_{4}\left(2 K^{\prime} v\right)=4 \pi \tilde{q}^{2} \sin 2 \pi v+\ldots
\end{aligned}
$$

We have also to use the relation $\frac{2 K}{A K^{\prime}} \rightarrow \frac{1}{2 \pi}$, which follows from Eq. (13) in the $K \rightarrow \infty$ limit. Then, we find

$$
\begin{aligned}
& W_{\mathrm{sc}}\left(A_{1}, A_{2}\right)=-i \pi a \oint \frac{d v}{2 \pi i} e^{i \frac{\pi a A_{2}}{2 K^{\prime}} \cot \pi v+2 \pi i v} \\
& \left\{1-4 \tilde{q}+i \frac{2 \pi a A_{1}}{K^{\prime}} \tilde{q}^{2} \sin 2 \pi v-4 \tilde{q}^{2}\left(\cos 2 \pi v-\sin ^{2} 2 \pi v\right)+\ldots\right\}= \\
& -\frac{1}{2} \oint \frac{d x}{2 \pi i} \frac{x-1}{x+1} e^{\frac{\pi a A_{2}}{2 K^{\prime}} x}\left\{1+4 \tilde{q}^{2}+8 \tilde{q}^{2} \log \tilde{q}-2 A_{1} \tilde{q}^{2} \frac{x}{x^{2}-1}-\right. \\
& \left.4 \tilde{q}^{2}\left[\frac{x^{2}+1}{x^{2}-1}+\left(\frac{2 x}{x^{2}-1}\right)^{2}\right]+\ldots\right\}
\end{aligned}
$$

The integral is reduced to residues at $x=1$ and $x=-1$ and we find

$$
W_{\mathrm{sc}}\left(A_{1}, A_{2}\right)=e^{-\frac{A_{2}}{2}}+e^{-\frac{A_{1}}{2}}+\left(-1+3 A_{2}+\frac{A_{2}^{2}}{2}\right) e^{-\frac{2 A_{2}+A_{1}}{2}}+\ldots
$$

As the points 0 and $2 K$ are separated in the $K \rightarrow \infty$ limit by the infinite distance, we have the non-symmetric expansion. If we shifted the variable $z \rightarrow z+2 K$ before making the modular transformation, we would find the answer with permuted $A_{1}$ and $A_{2}$. 
The first look at the expansion (52) shows that it is compatible with the sum-over-branched-coverings interpretation. The first terms show the standard $Q C D_{2}$ area low. The last one represents the simplest non-trivial covering with a disc. Nevertheless, there is some puzzle here which reads: "How can the property $W(0, A)=W(A, 0)=1$ be agreed with an openstring picture, we would like to have?".

\section{Acknowledgments}

The discussions with V.A.Kazakov have very much contributed to this work. I would like to thank B.Rusakov for the numerous stimulating discussions and kind hospitality at Physics Department of the Tel-Aviv University, where this work was started.

Note added: After having completed the manuscript, I learned that Eq. (27) has also been obtained by Jean-Mark Daul and Volodya Kazakov [10]. 


\section{References}

[1] D.Gross, Nucl. Phys. B400 (1993) 161; D.Gross and W.Taylor, Nucl. Phys. B400 (1993) 181; preprint PUPT-1382 (March, 1993).

[2] I.K.Kostov, Nucl. Phys. B256 (1986) 86; and Saclay preprint S.Ph.T93-060 (1993).

[3] A.A.Migdal, Sov. Phys. JETP 42 (1975) 413, 743.

[4] B.Rusakov, Mod. Phys. Lett. A5 (1990) 693.

[5] M.Douglas and V.A.Kazakov, Large $N$ phase transition in continuum QC $D_{2}$, preprint LPTENS-93/20, RU-93-17 (May, 1993).

[6] B.Rusakov, Phys. Lett. B303 (1993) 95.

[7] V.A.Kazakov and I.K.Kostov, Nucl. Phys. B176 (1980) 199; Phys. Lett. B105 (1981) 453; V.A.Kazakov, Nucl. Phys. B179 (1981) 283.

[8] B.Rusakov, to be published.

[9] A.Erdélyi, Higher Transcendental Functions, Vol. 2, McGraw-Hill (1953).

[10] J.-M.Daul and V.Kazakov, Wilson loop for large N QCD on the sphere, preprint LPTENS 93/37 (October, 1993) 